\title{
Using nutation-frequency-selective pulses to reduce radio-frequency field inhomogeneity in solid-state NMR
}

\author{
Kathrin Aebischer ${ }^{1}$, Nino Wili ${ }^{1}$, Zdeněk Tošner $^{2}$, and Matthias Ernst ${ }^{1}$ \\ ${ }^{1}$ Physical Chemistry, ETH Zürich, Vladimir-Prelog-Weg 2, 8093 Zürich, Switzerland \\ ${ }^{2}$ Department of Chemistry, Faculty of Science, Charles University, Hlavova 8, 12842 Prague 2, Czech Republic
}

Correspondence: Matthias Ernst (maer@ethz.ch)

Received: 15 July 2020 - Discussion started: 21 July 2020

Revised: 20 August 2020 - Accepted: 27 August 2020 - Published: 9 September 2020

\begin{abstract}
Radio-frequency (rf) field inhomogeneity is a common problem in NMR which leads to non-ideal rotations of spins in parts of the sample. Often, a physical volume restriction of the sample is used to reduce the effects of rf-field inhomogeneity, especially in solid-state NMR where spacers are inserted to reduce the sample volume to the centre of the coil. We show that band-selective pulses in the spin-lock frame can be used to apply $B_{1}$-field selective inversions to spins that experience selected parts of the rf-field distribution. Any frequency band-selective pulse can be used for this purpose, but we chose the family of I-BURP pulses (Geen and Freeman, 1991) for the measurements demonstrated here. As an example, we show that the implementation of such pulses improves homonuclear frequency-switched Lee-Goldburg decoupling in solid-state NMR.
\end{abstract}

1

\section{Introduction}

Radio-frequency (rf) inhomogeneity is one of the experimental imperfections in solid-state NMR experiments that is almost unavoidable and often leads to deterioration of the performance of pulse sequences. Especially in small magicangle spinning (MAS) probes used in high-resolution solidstate NMR, where the solenoid coil is close to the sample space, significant rf-field inhomogeneity is observed over the sample volume (Tosner et al., 2017). The magnitude of the rf-field inhomogeneity can be characterized by a simple nutation experiment. Characterizing the full spatial distribution of the rf-field amplitude over a rotor requires single- or triple-axis gradients for imaging (Guenneugues et al., 1999), but simpler methods using the $z$ shim have also been proposed (Odedra and Wimperis, 2013). Alternatively, the rffield distribution over the rotor can be measured by a ballshift measurement (Paulson et al., 2004) or calculated using numerical simulations of the coil and rf circuit (Tosner et al., 2017, 2018). Simulations and measurements show that typical MAS solid-state NMR probes have large rf-field distributions along the rotor axis and in addition along the radial dimension. Such rf-field inhomogeneity often manifests itself in the spectrum as reduced signal intensity in polarizationtransfer experiments (Nishimura et al., 2001), in broadened lines in decoupling experiments (Vega, 2004), or in spatial selectivity in cross-polarization experiments (Gupta et al., 2015).

Experimentally reducing the rf-field inhomogeneity is often achieved through sample restriction by inserting spacers into the upper and lower parts of the rotor and filling the sample in the central part of the rotor. However, since there is also significant radial rf-field inhomogeneity (Tosner et al., 2017) in MAS NMR probes, even very thin slices of samples still show a significant distribution of rf-field amplitudes. Sometimes, even spherically restricted samples are used inside the cylindrical rotors, especially in high-resolution MAS (Lindon et al., 2009). In principle, magnetic-field gradients also allow the restriction of the sample space (Charmont et al., 2000) along the axis of the rotor if a magic-angle gradient is used. As there are very few probes in solid-state NMR that have gradient capability, this approach is not suitable for widespread application.

Alternatively, radio-frequency field selective pulses can be used to achieve sample restriction. This was demonstrated some years ago; however, the numerically optimized radio- 
frequency selective pulses showed many sidebands, especially in the low rf-field region (Charmont et al., 2002). In this publication, we propose a simpler approach to implement amplitude selective pulses in the spin-lock frame by using a modulation of the pulses that is resonant with the spin-lock field. In such an implementation, any frequencyselective pulses (Emsley, 2007) that have been designed for chemical-shift selection can be used. In our work, we have chosen the I-BURP class of band-selective inversion pulses (Geen and Freeman, 1991) that shows a very sharp inversion profile. Similar approaches using resonant rf irradiation in nested rotating frames have been reported before in liquid-state NMR (Grzesiek and Bax, 1995), in recoupling experiments in solid-state NMR (Khaneja and Nielsen, 2008; Straasø et al., 2009), and in EPR (Wili et al., 2020).

\section{Theory}

In NMR, radio-frequency pulses are implemented as resonant $\mathrm{rf}$ irradiation orthogonal to the static magnetic field that leads to a static magnetic field in the rotating frame (Ernst et al., 1990). The Hamiltonian in the laboratory frame is given by

$$
H(t)=H_{\mathrm{Z}}+H_{\mathrm{rf}}(t)=\omega_{0} I_{z}+2 \omega_{1}(t) \cos \left(\omega_{\mathrm{rf}} t+\varphi(t)\right) I_{x},
$$

the sum of the Zeeman interaction and the time-dependent radio-frequency term describing a linear-polarized resonant radio-frequency irradiation. After transformation to the usual rotating frame (with the modulation frequency of the radio frequency), we obtain two terms. One has a zero frequency and is static in the rotating frame, while the second one rotates at twice the frequency and is usually neglected. It can, however, give rise to Bloch-Siegert effects (Bloch and Siegert, 1940). Neglecting the counter-rotating part, we obtain a first-order rotating-frame Hamiltonian of the form

$$
H^{\prime}(t)=\left(\omega_{0}-\omega_{\mathrm{rf}}\right) I_{z}+\omega_{1}(t)\left(\cos \varphi(t) I_{x}+\sin \varphi(t) I_{y}\right) .
$$

Note that the factor of 2 in front of $\omega_{1}(t)$ in Eq. (1) is just introduced for convenience to obtain an amplitude of $\omega_{1}(t)$ in the rotating frame. Application of circular-polarized rf is also possible and would eliminate this factor of 2 but is rarely implemented in NMR. Using an appropriate phase or amplitude modulation will allow us to implement frequency-band selective pulses in the normal rotating frame (Emsley, 2007). Under the condition that $\omega_{0}-\omega_{\mathrm{rf}}$ is small (near-resonance irradiation), we can now apply a strong spin-lock field along the $x$ axis and use an orthogonal resonant field along the $y$ axis to apply pulses in the spin-lock frame. Assuming $\omega_{0}=\omega_{\mathrm{rf}}$, the Hamiltonian for such an irradiation in the usual rotating frame would be

$$
H^{\prime}(t)=\omega_{1} I_{x}+2 \omega_{2}(t) \cos \left(\omega_{\bmod } t\right) I_{y} .
$$

Note the similarity of Eqs. (3) and (1). Transforming into an interaction frame with $\omega_{\bmod } I_{x}$ and neglecting again the counter-rotating part leads to a first-order interaction-frame Hamiltonian of the form

$H^{\prime \prime}(t)=\left(\omega_{1}-\omega_{\bmod }\right) I_{x}+\omega_{2}(t) I_{y}$,

where $\omega_{1}$ might be broadly distributed due to rf-field inhomogeneity. We can now choose $\omega_{2}(t)$ such that it corresponds to a frequency-band selective pulse and set $\omega_{\bmod }=$ $\omega_{1}$ to obtain a radio-frequency field selective pulse, i.e. a pulse that is selective in $\omega_{1}$. Again, we can implement any arbitrarily shaped pulse that was designed for band selection in the chemical-shift space (Emsley, 2007). Figure 1 illustrates how such a selective pulse in the spin-lock frame is implemented for the example of an I-BURP-2 pulse (Geen and Freeman, 1991) that has been used in our experiments.

By changing the modulation frequency, we can select different parts of the rf-field distribution in the probe, and by changing the pulse length we can adjust the width of the selected region. The bandwidth of the I-BURP-2 pulse is roughly $4 / \tau_{\mathrm{p}}$, where $\tau_{\mathrm{p}}$ is the length of the pulse. The pulse shown in Fig. 1 with $\tau_{\mathrm{p}}=200 \mu \mathrm{s}$ has, therefore, an excitation bandwidth of approximately $20 \mathrm{kHz}$. If the amplitude of the pulse in the spin-lock frame is not much lower than the amplitude of the spin lock itself (very broad excitation bandwidth), Bloch-Siegert-type (Bloch and Siegert, 1940) phenomena might become visible. In this case, a pulse using circular polarized irradiation in the spin-lock frame can, in principle, be used to avoid these problems.

\section{Numerical simulations}

Numerical simulations of the performance of the I-BURP2 pulses in the spin-lock frame were carried out using the GAMMA spin-simulation environment (Smith et al., 1994). Sweeping the spin-lock field from 10 to $150 \mathrm{kHz}$, using a modulation frequency of $100 \mathrm{kHz}$ and a pulse length of $1 \mathrm{~ms}$ (Fig. 2a) and $200 \mu \mathrm{s}$ (Fig. 2b), respectively, we have simulated the inversion efficiency of the spin-locked magnetization. Figure 2 shows that the bandwidth is indeed roughly $4 \mathrm{kHz}$ (Fig. 2a) and $20 \mathrm{kHz}$ (Fig. 2b), and outside this band there are only very small artefacts visible. The line in black shows the profile assuming an ideal rf-field amplitude for the I-BURP-2 pulse $\left(\nu_{2}=1 / \tau_{\mathrm{p}}\right)$ corresponding to a perfect inversion pulse independent of the value of $\nu_{1}$, while for the blue line the rf-field amplitude was scaled $\left(v_{2}=\left(1 / \tau_{\mathrm{p}}\right)\left(v_{1} / v_{\text {mod }}\right)\right)$, leading to flip-angle deviation in the inversion pulse if the spin-lock amplitude $\left(v_{1}\right)$ is different from the modulation frequency $\left(v_{\bmod }\right)$. There are only small differences between the two simulations, indicating a good compensation of rf-field amplitude errors in the I-BURP-2 pulse (Geen and Freeman, 1991). This clearly shows the excellent radio-frequency amplitude selectivity of the I-BURP2 pulse in the spin-lock frame. Changing the pulse length will allow us to adjust the bandwidth of the selected area. In principle, any selective pulse used in NMR can be implemented in this scheme. 


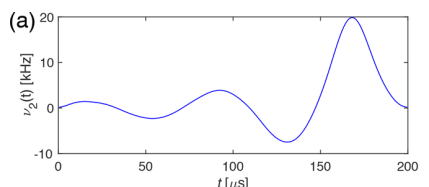

(b)

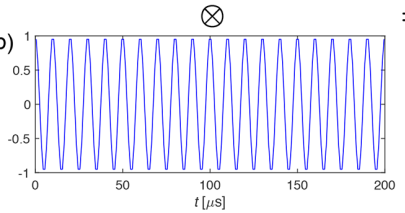

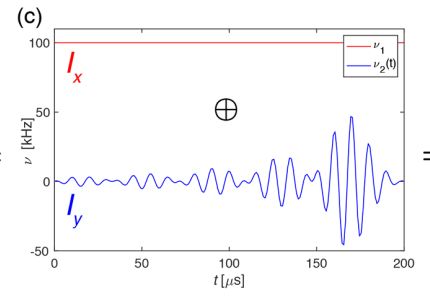
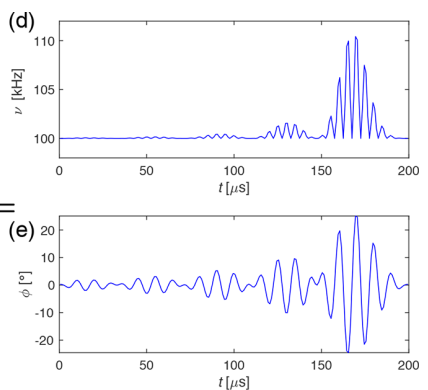

Figure 1. Generating the I-BURP-2 pulse in the spin-lock frame with a $100 \mathrm{kHz}$ spin-lock field, $200 \mu$ s pulse length, $100 \mathrm{kHz}$ modulation frequency and time resolution of $1 \mu$ s. (a) Pulse shape of the I-BURP-2 pulse which is multiplied by the (b) frequency modulation and leads to the (c) $y$ component (blue) of the rf field. The spin lock is the $x$ component (red) of the rf field. The two components are combined and result in (d) the amplitude and (e) phase of the final pulse shape that can be used in the experiment. The time resolution for the shapes was usually set to $1 \mu \mathrm{s}$.
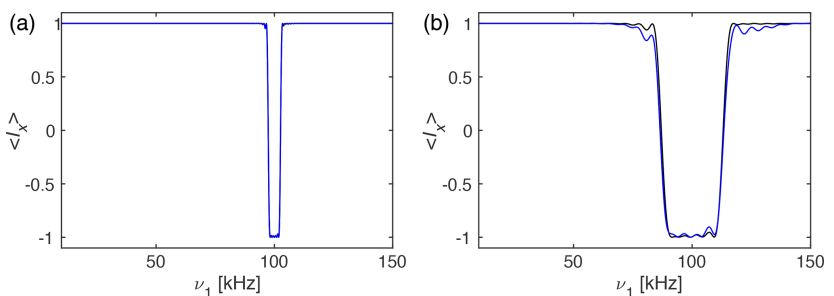

Figure 2. Plot of the expectation value $\left\langle I_{x}\right\rangle$ as a function of the rffield amplitude $\nu_{1}$ in the range of 10 to $150 \mathrm{kHz}$ under an I-BURP-2 pulse in the spin-lock frame using ideal rf-field amplitudes (black) and scaled rf-field amplitudes (blue). The modulation frequency was set to $v_{\text {mod }}=100 \mathrm{kHz}$ and the pulse had a length of (a) $1 \mathrm{~ms}$ and (b) $200 \mu$ s corresponding to an inversion range of about 4 and $20 \mathrm{kHz}$, respectively. One can clearly see the inversion band around the $100 \mathrm{kHz}$ modulation frequency, with virtually no artefacts outside the inversion band. The black line corresponds to an ideal rffield amplitude of the I-BURP-2 pulse of $\nu_{2}=1 / \tau_{\mathrm{p}}$, while the blue line uses $v_{2}=\left(1 / \tau_{\mathrm{p}}\right)\left(v_{1} / v_{\text {mod }}\right)$, as would be the case in a real experiment with rf-field inhomogeneity. Simulations using circularpolarized radio-frequency fields that address the role of possible Bloch-Siegert effects can be found in Fig. S05.

\section{Experimental results and discussion}

The experimental implementation of such pulses was tested by combining an I-BURP-2 inversion pulse in the spinlock frame with a two-dimensional nutation experiment. A schematic representation of the pulse sequence is shown in Fig. 3. The I-BURP-2 pulse preceding the $t_{1}$ nutation period leads to a band-selective inversion of the magnetization in the spin-lock frame and will thus invert parts of the nutation spectrum. The length of the spin lock was always set to the length of the I-BURP-2 pulse $\left(\tau_{\mathrm{p}}\right)$. The position of the inverted part is determined by the modulation frequency $\omega_{\bmod }$ of the I-BURP-2 pulse, and its bandwidth can be adjusted by changing the length of the inversion pulse. Difference spectra can be obtained by combining consecutive scans with and

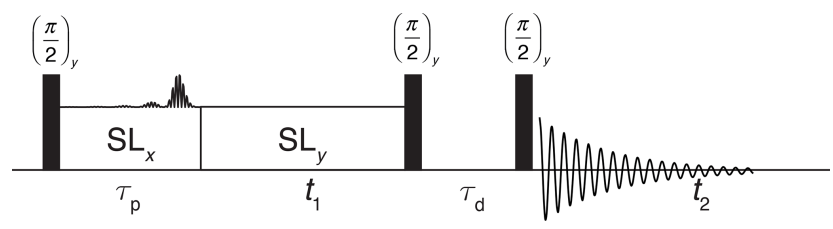

Figure 3. Schematic representation of the pulse sequence used for testing of the inversion properties of the I-BURP-2 pulse in the spin-lock frame. After the initial $90^{\circ}$ pulse, the magnetization is spin locked along $x$ and the modulated I-BURP-2 inversion pulse applied along $y$. During the subsequent $t_{1}$ time the magnetization nutates about the field along $y$. To obtain pure-phase spectra, a $z$ filter with a dephasing delay is used to select a single component after the nutation. Difference spectra can be obtained by replacing the I-BURP-2 pulse in the spin-lock frame with a simple spin lock in alternating scans while shifting the receiver phase by $180^{\circ}$.

without the inversion pulse and using an appropriate phase cycle on the receiver phase which is shifted by $180^{\circ}$.

Figure 4 shows nutation spectra of adamantane spinning at $20 \mathrm{kHz}$ MAS frequency recorded at a proton resonance frequency of $500 \mathrm{MHz}$ using a Bruker $1.9 \mathrm{~mm}$ MAS probe which has a relatively narrow $\mathrm{rf}$-field inhomogeneity profile. The rf-field amplitude corresponds to the zero crossing of a $5 \mu \mathrm{s} \pi$ pulse (indicative of a $100 \mathrm{kHz}$ rf-field amplitude) and reaches its maximum around $109 \mathrm{kHz}$ (blue line). Preceding the nutation by an I-BURP-2 pulse in the spin-lock frame inverts part of the nutation spectrum, as can be seen from the green and red lines in Fig. 4. The pulses were generated assuming a $100 \mathrm{kHz}$ rf-field amplitude based on the pulselength determination, illustrating the good compensation of the I-BURP-2 pulses for amplitude mis-setting. The inverted region narrows with increasing pulse length $\left(\tau_{\mathrm{p}}=400 \mu \mathrm{s}\right.$ in Fig. $4 \mathrm{a}, \tau_{\mathrm{p}}=800 \mu \mathrm{s}$ in Fig. $4 \mathrm{~b}, \tau_{\mathrm{p}}=1600 \mu \mathrm{s}$ in Fig. $4 \mathrm{c}$ ) and is shifted with a change in the modulation frequency from 90 to $100 \mathrm{kHz}$ (red and green lines in Fig. 4). Virtually no artefacts outside the desired inversion range are visible. At slower spinning frequencies, MAS sidebands of the inver- 

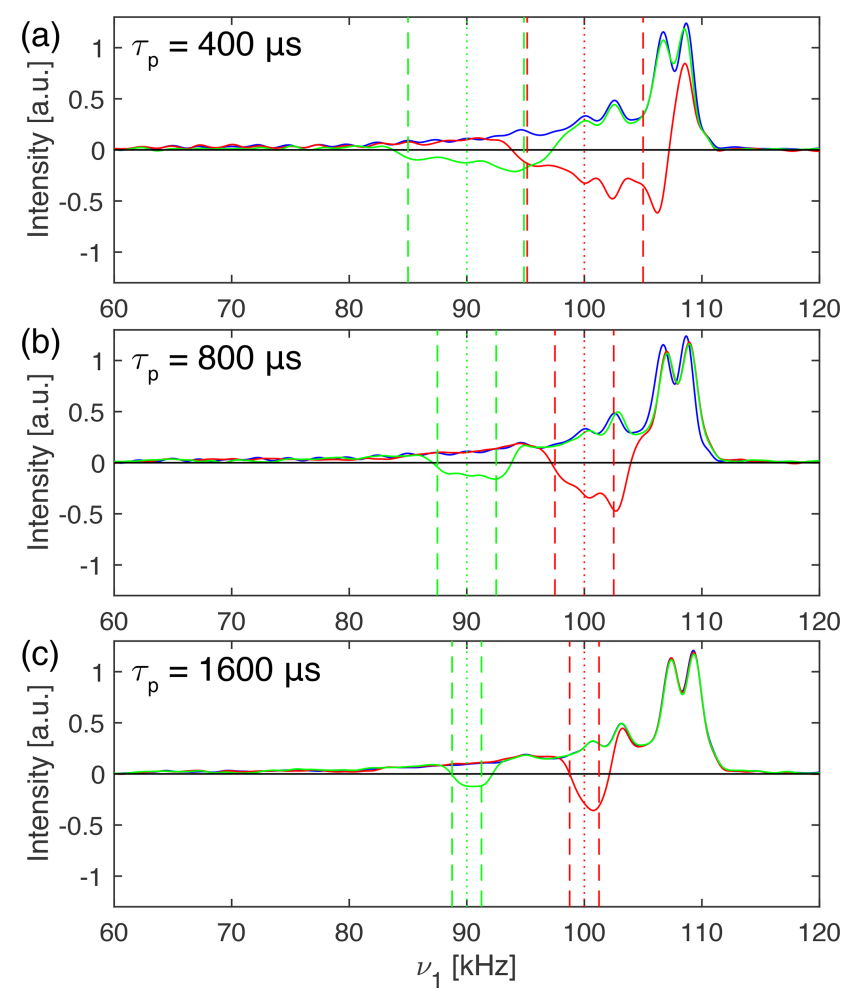

Figure 4. Proton nutation spectra of adamantane spinning at $20 \mathrm{kHz}$ at $500 \mathrm{MHz}$ proton resonance frequency using a Bruker $1.9 \mathrm{~mm}$ MAS probe. In blue, a standard nutation experiment is shown with a nominal rf-field amplitude of $100 \mathrm{kHz}$ determined by the zero crossing of a $5 \mu \mathrm{s} \pi$ pulse. The nutation spectra in green and red were preceded by an I-BURP-2 pulse of length (a) $400 \mu \mathrm{s}$, (b) $800 \mu \mathrm{s}$ and (c) $1600 \mu \mathrm{s}$ using a modulation frequency of $90 \mathrm{kHz}$ (green) and $100 \mathrm{kHz}$ (red), respectively. The fact that the inversion ranges are slightly shifted compared to the theoretical ones (indicated by dashed and dotted lines) is most likely due to fluctuations in the amplifier gain, as can be seen from Fig. S03.

sion profile become visible (see Figs. S01 of the Supplement). In comparison to the width and position of the theoretical inversion profiles indicated by dashed (borders) and dotted (centre) lines, the experimental inversion profiles are shifted to slightly higher rf amplitudes. A similar experiment on a $600 \mathrm{MHz}$ spectrometer showed no such deviations (Fig. S01). This shift is most likely due to fluctuations in the amplifier gain which is shown in detail in Fig. S03. Adding a 2 ms spin-lock pulse before the selection pulse (see Fig. S02 for the pulse scheme) leads to a shift of the maximum of the nutation frequency by about $1-2 \mathrm{kHz}$. This temporal instability of the gain of the radio-frequency amplifiers depends on the exact setting of the radio frequency, the output power of the amplifier, and the pulse history. The amplification factor can slowly drift up or down over time, leading to differences in the selected rf-field amplitudes and the measured ones in the nutation experiment.
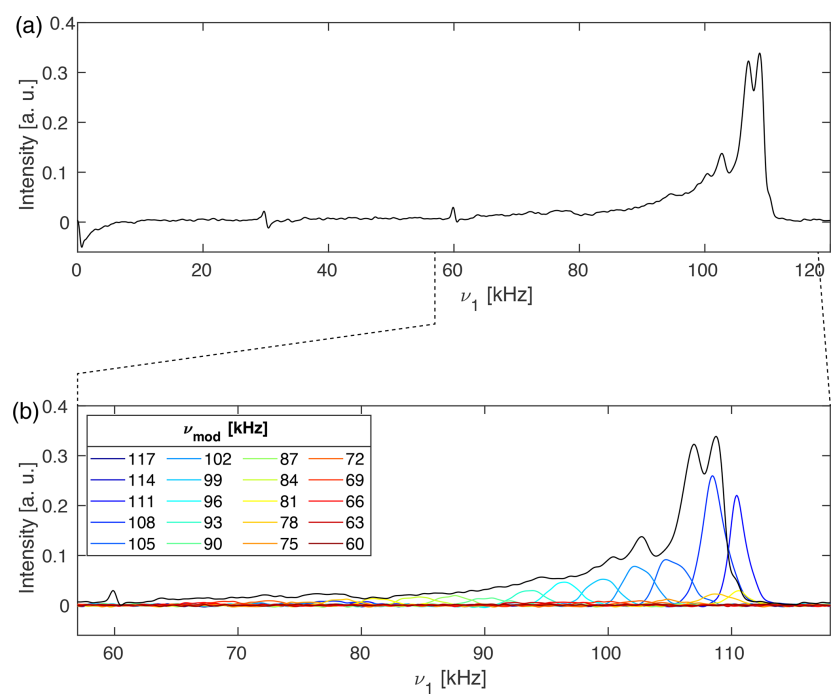

Figure 5. (a) ${ }^{1} \mathrm{H}$ nutation spectra of natural-abundance glycine measured at a proton-resonance frequency of $500 \mathrm{MHz}$ spinning at $30 \mathrm{kHz}$ in a Bruker $1.9 \mathrm{~mm}$ outer-diameter rotor which was completely filled. The nutation spectrum without selective inversion (black) shows MAS modulation bands at 30 and $60 \mathrm{kHz}$ due to the MAS-induced time dependence of amplitude and phase of the rf irradiation. (b) Expanded region of (a) overlaid in colour with various difference spectra using a 2 ms I-BURP-2 pulse in the spinlock frame where the modulation frequency was moved through the width of the nutation spectrum. Contributions that lie outside the nutation spectrum are due to the drift of the radio-frequency amplifier gain over the course of different experiments. To allow a more detailed view of the various sub-spectra in (b), they are also shown in a separated plot in Fig. S04.

We can now combine a nutation spectrum with a preceding band-selective inversion pulse with a nutation spectrum where the I-BURP-2 pulse in the spin-lock frame is replaced with a simple spin lock and the receiver phase is shifted by $180^{\circ}$ in alternating scans to obtain $B_{1}$-field selective nutation spectra. Thus, difference spectra are obtained where only certain regions of the rf-field amplitude are selected by the pulse in the spin-lock frame. This allows the reduction of the rf-field inhomogeneity over the sample at the cost of lowering the signal intensity. In essence, this is a restriction of the sample not in terms of physical location, but in terms of the rf field experienced by certain crystallites in the rotor.

Figure 5a shows a normal proton nutation spectrum of glycine at a spinning speed of $30 \mathrm{kHz}$. The spectra were recorded at a proton resonance frequency of $500 \mathrm{MHz}$ using a Bruker $1.9 \mathrm{~mm}$ MAS probe. The nominal rf-field amplitude was calibrated by the zero crossing of a $5 \mu$ s $\pi$ pulse. In Fig. 5b amplitude-selected difference spectra are overlaid over an expanded region of the nutation spectrum where the modulation frequency of the inversion pulse in the spin-lock frame was shifted through the width of the nutation spectrum. 


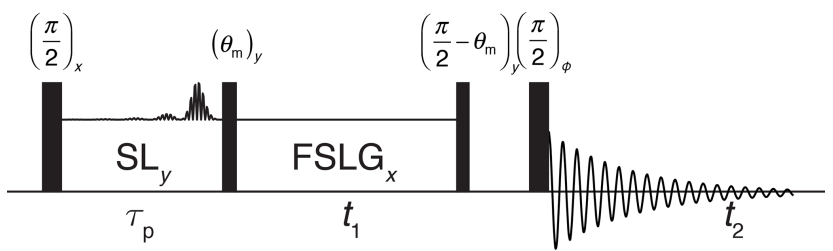

Figure 6. Schematic representation of the pulse sequence used to acquire two-dimensional proton-proton chemical-shift correlation spectra with high resolution through FSLG decoupling achieved in $t_{1}$. The final $90^{\circ}$ pulse is phase cycled together with the receiver through all four quadrature phases. States-type phase-sensitive detection in the indirect dimension is implemented by shifting the phase of the first $90^{\circ}$ pulse and the spin lock by $90^{\circ}$. To select parts of the sample by the I-BURP-2 pulse in the spin-lock frame, two scans with and without the inversion pulse were subtracted by shifting the receiver phase by $180^{\circ}$.

The nutation spectrum without selective inversion (black line) has a maximum at $108 \mathrm{kHz}$ and shows MAS modulation bands at 30 and $60 \mathrm{kHz}$ that appear due to the MAS-induced time dependence of the rf amplitude and phase. Nutation experiments with preceding I-BURP-2 pulses with a length of $2 \mathrm{~ms}$ and modulation frequencies spanning the width of the nutation spectrum are shown in colour in Fig. $5 \mathrm{~b}$ for an expanded region of the nutation spectrum. It can be seen that the experiment allows the selection of different parts of the rf-field distribution depending on the modulation frequency that is chosen. Interestingly, none of the sub-spectra shows the MAS modulation bands at 30 and $60 \mathrm{kHz}$. As these modulation bands stem from areas within the sample volume experiencing large rf amplitude and phase modulations, dephasing of the magnetization in those regions during spin lock could be responsible for their disappearance. However, this is not yet fully understood and requires a more careful investigation.

To showcase the potential of using rf-field amplitude selective pulses to reduce the effects of $\mathrm{rf}$ inhomogeneity in experiments, homonuclear-decoupled proton spectra under MAS using frequency-switched Lee-Goldburg (FSLG) irradiation (Bielecki et al., 1989, 1990; Mote et al., 2016) were recorded. FSLG decoupled spectra were acquired in the indirect dimension of a two-dimensional correlation experiment with direct proton detection under MAS. The pulse sequence used for these experiments is shown in Fig. 6. All spectra were acquired with the basic FSLG sequence without any super cycling.

Spectra with and without a $B_{1}$-field selection were acquired at $14 \mathrm{kHz}$ MAS and are shown in Fig. 7 for glycine (Fig. 7a), the dipeptide $\beta$-Asp-Ala (Fig. 7b), and L-histidine (Fig. 7c) at a proton resonance frequency of $500 \mathrm{MHz}$ using a Bruker $1.9 \mathrm{~mm}$ MAS probe. The nominal rf-field amplitude for hard pulses and during spin lock was set to $100 \mathrm{kHz}$ using a nutation experiment for the calibration. The carrier was placed outside the spectral region of interest, but

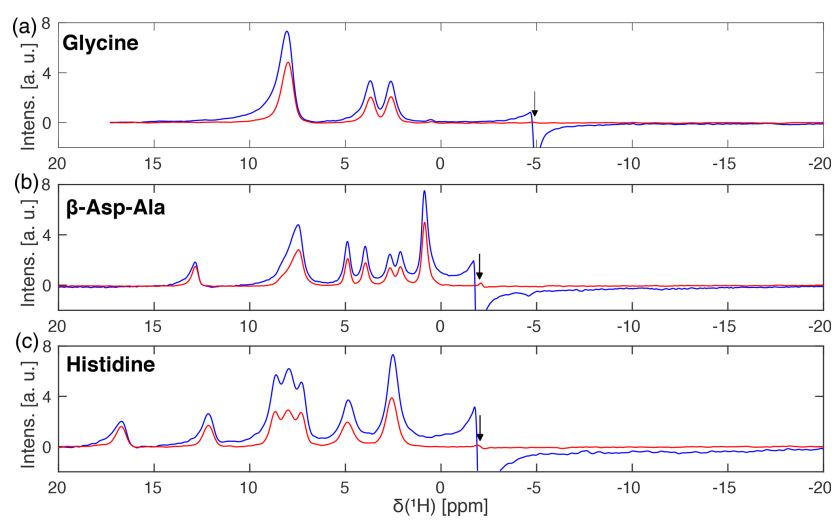

Figure 7. Frequency-switched Lee-Goldburg decoupled proton spectra of (a) natural-abundance glycine, (b) natural-abundance $\beta$-Asp-Ala and (c) natural-abundance L-histidine with (red) and without (blue) a $800 \mu$ s I-BURP-2 pulse used to select the part of the rotor experiencing rf-field amplitudes close to the nominal value of $100 \mathrm{kHz}$. Spectra were recorded at a proton resonance frequency of $500 \mathrm{MHz}$ using a $1.9 \mathrm{~mm}$ Bruker MAS probe (completely filled) spinning the sample at $14 \mathrm{kHz}$. FSLG decoupling was achieved using a continuous phase ramp with a time resolution of $50 \mathrm{~ns}$ and an effective field of $125 \mathrm{kHz}$. The frequency axes are scaled by the experimentally determined scaling factors of (a) 0.562 , (b) 0.557 , and (c) 0.572 , which are very close to the theoretical one of $\cos \left(\theta_{\mathrm{m}}\right)=\sqrt{1 / 3} \approx 0.577$. Arrows indicate the position of the carrier frequency. Narrower lines and a strongly reduced zero-frequency artefact are observed for spectra with a $B_{1}$-field selection.

no experimental optimization of its exact position was performed. Figure 7 clearly shows that spectra recorded with a rf-field selective $800 \mu$ I-BURP-2 pulse in the spin-lock frame with a modulation frequency of $100 \mathrm{kHz}$ (red lines) have clearly narrower lines (see Table 1) than FSLG spectra acquired without the selective inversion (blue lines). Moreover, the zero-frequency artefact at the position of the carrier frequency is eliminated, and the foot on the left-hand side of the peaks due to a distribution of the isotropic chemical-shift scaling factors (Hellwagner et al., 2020) is reduced. Since the band-selective inversion pulse only selects regions within the sample space experiencing rf-field amplitudes close to $100 \mathrm{kHz}$, the selection also leads to a decrease in signal intensity roughly by a factor of 2 in the integrated peak intensity of Fig. 7. For some of the peaks, the peak height is reduced significantly less due to the elimination of the broad components on the left-hand side of the peaks. The experimentally determined chemical shift scaling factors (see figure caption) are very close to the theoretical value for FSLG decoupling given by $\cos \left(\theta_{\mathrm{m}}\right)=\sqrt{1 / 3} \approx 0.577$.

The significant reduction of the carrier-frequency artefact permits the recording of spectra where the carrier is positioned in the centre of the spectral region of interest. This allows a substantial reduction of the spectral width and faster data acquisition in the two-dimensional schemes used here. 
Table 1. Full width at half maximum (FWHM) for homonuclear decoupled proton spectra at $500 \mathrm{MHz}$ shown in Fig. 7.

\begin{tabular}{|c|c|c|c|c|c|c|}
\hline \multirow{4}{*}{$\begin{array}{l}\text { Sample } \\
\text { Glycine }\end{array}$} & \multirow{4}{*}{$\begin{array}{r}\text { Figure } \\
7 \mathrm{a}\end{array}$} & \multirow{2}{*}{$\begin{array}{r}\begin{array}{r}\delta\left({ }^{1} \mathrm{H}\right) \\
(\mathrm{ppm})\end{array} \\
2.62\end{array}$} & \multicolumn{2}{|c|}{$\begin{array}{c}\text { FWHM }^{*} \\
\text { (standard FSLG) }\end{array}$} & \multicolumn{2}{|c|}{$\begin{array}{c}\text { FWHM }^{*} \\
(800 \mu \text { s I-BURP-2) }\end{array}$} \\
\hline & & & $180 \mathrm{~Hz}$ & $0.64 \mathrm{ppm}$ & $160 \mathrm{~Hz}$ & $0.58 \mathrm{ppm}$ \\
\hline & & 3.68 & $190 \mathrm{~Hz}$ & $0.68 \mathrm{ppm}$ & $160 \mathrm{~Hz}$ & $0.59 \mathrm{ppm}$ \\
\hline & & 8.00 & $240 \mathrm{~Hz}$ & $0.86 \mathrm{ppm}$ & $200 \mathrm{~Hz}$ & $0.73 \mathrm{ppm}$ \\
\hline \multirow{5}{*}{$\beta$-Asp-Ala } & \multirow{5}{*}{$7 b$} & 0.86 & $110 \mathrm{~Hz}$ & $0.41 \mathrm{ppm}$ & $90 \mathrm{~Hz}$ & $0.33 \mathrm{ppm}$ \\
\hline & & 3.95 & $110 \mathrm{~Hz}$ & $0.41 \mathrm{ppm}$ & $100 \mathrm{~Hz}$ & $0.34 \mathrm{ppm}$ \\
\hline & & 4.88 & $100 \mathrm{~Hz}$ & $0.37 \mathrm{ppm}$ & $90 \mathrm{~Hz}$ & $0.32 \mathrm{ppm}$ \\
\hline & & 7.45 & $260 \mathrm{~Hz}$ & $0.93 \mathrm{ppm}$ & $220 \mathrm{~Hz}$ & $0.78 \mathrm{ppm}$ \\
\hline & & 12.84 & $130 \mathrm{~Hz}$ & $0.48 \mathrm{ppm}$ & $100 \mathrm{~Hz}$ & $0.37 \mathrm{ppm}$ \\
\hline \multirow{4}{*}{ L-Histidine } & \multirow{4}{*}{$7 \mathrm{c}$} & 2.55 & $210 \mathrm{~Hz}$ & $0.73 \mathrm{ppm}$ & $190 \mathrm{~Hz}$ & $0.67 \mathrm{ppm}$ \\
\hline & & 4.91 & $240 \mathrm{~Hz}$ & $0.85 \mathrm{ppm}$ & $210 \mathrm{~Hz}$ & $0.73 \mathrm{ppm}$ \\
\hline & & 12.21 & $210 \mathrm{~Hz}$ & $0.72 \mathrm{ppm}$ & $170 \mathrm{~Hz}$ & $0.60 \mathrm{ppm}$ \\
\hline & & 16.79 & $230 \mathrm{~Hz}$ & $0.80 \mathrm{ppm}$ & $170 \mathrm{~Hz}$ & $0.60 \mathrm{ppm}$ \\
\hline
\end{tabular}

* Values given in $\mathrm{Hz}$ are taken from the processed and unscaled spectra. Values in ppm include the isotropic chemical-shift scaling and correspond to the values in the plotted spectra.

Resulting FSLG decoupled spectra of L-histidine with different pulse lengths for the I-BURP-2 selection pulse acquired at a proton resonance frequency of $600 \mathrm{MHz}$ using a $2.5 \mathrm{~mm}$ Bruker MAS probe spinning the sample at $14 \mathrm{kHz}$ are shown in Fig. 8. Increasing the length of the I-BURP-2 pulse and thus improving the rf-field selectivity (narrower bandwidth) leads to a decrease in linewidth and a reduction of the foot of the lines pointing away from the carrier frequency which is due to the distribution of isotropic chemical-shift scaling factors (Hellwagner et al., 2020). However, the achieved line narrowing using more selective pulses again coincides with lower signal intensity (see Table 2 for numerical values of the linewidth). The intensity of the signals drops significantly by a factor of 2 to 3 when going from 200 or $400 \mu$ s pulse length to a much more selective $800 \mu$ s or 2 ms pulse length. Figure 8 also illustrates that the use of $B_{1}$-field selective pulses allows the placement of the carrier frequency inside the region of interest, as only a small but clearly visible carrier-frequency artefact is observed which can be completely eliminated by placing the carrier outside the region of interest (Fig. 8, blue spectrum). The magnitude of the carrierfrequency peak increases with increasing proximity to a real spectral peak. Again, the chemical-shift scaling factors (see figure caption for numerical values) are very close to the theoretical value.

\section{Materials and methods}

Numerical simulations were implemented using the GAMMA spin-simulation environment (Smith et al., 1994). All simulations were performed using a single-spin system and time slicing of the time-dependent Hamiltonian to calculate the time evolution of the density operator in the standard rotating frame.
All powdered samples used in the measurements (adamantane, natural-abundance glycine, natural-abundance L-histidine $\cdot \mathrm{HCl} \cdot \mathrm{H}_{2} \mathrm{O}$, natural-abundance $\beta$-Asp-Ala) were purchased from commercial sources and used without further purification. Rotors were filled completely and the sample space was not spatially restricted.

Experiments were carried out on Bruker Avance III HD NMR spectrometers, operating at a proton resonance frequency of $500 \mathrm{MHz}(600 \mathrm{MHz})$, equipped with a Bruker $1.9 \mathrm{~mm}(2.5 \mathrm{~mm})$ triple-resonance probe (in doubleresonance configuration) at a temperature of $285 \mathrm{~K}$. The shaped pulses for the FSLG homonuclear decoupling and the I-BURP-2 pulses were programmed in Matlab (The Mathworks, Natick, MA) and exported to Bruker TopSpin shape file format using home-written routines.

Two-dimensional nutation spectra were acquired with a simple cosine modulation in $t_{1}$ at a spinning speed of $30 \mathrm{kHz}$ $(20 \mathrm{kHz})$ for adamantane (glycine) at a proton resonance frequency of $500 \mathrm{MHz}$. The spin-lock rf-field amplitude used for the I-BURP-2 pulses was set to $100 \mathrm{kHz}$ as determined by the zero crossing of a $5 \mu \mathrm{s} \pi$ pulse. Typically, 256-512 $t_{1}$ increments with eight scans each and a time increment of $3.5 \mu$ s, corresponding to a spectral width of $286 \mathrm{kHz}$, were recorded. The spectral width in $t_{2}$ was set to $100 \mathrm{kHz}$ and 1024 complex data points were acquired. Spectra were processed in Matlab using a cosine-squared apodization function. Example processing scripts are given in the Supplement.

Homonuclear decoupled proton spectra were acquired as two-dimensional ${ }^{1} \mathrm{H}-{ }^{1} \mathrm{H}$ correlation spectra with FSLG decoupling (Bielecki et al., 1989, 1990; Mote et al., 2016) in the indirect dimension using States-type data acquisition (States et al., 1982) for sign discrimination and phase-sensitive detection in the indirect dimension. Measurements were per- 
Table 2. Full width at half maximum (FWHM) for homonuclear decoupled proton spectra of L-histidine at $600 \mathrm{MHz}$ shown in Fig. 8 .

\begin{tabular}{|c|c|c|c|c|c|c|c|c|c|c|}
\hline \multirow{2}{*}{$\begin{array}{l}\delta\left({ }^{1} \mathrm{H}\right) \\
(\mathrm{ppm})\end{array}$} & \multicolumn{2}{|c|}{$\begin{array}{c}\mathrm{FWHM}^{*} \\
\tau_{\mathrm{p}}=200 \mu \mathrm{s}\end{array}$} & \multicolumn{2}{|c|}{$\begin{array}{c}\mathrm{FWHM}^{*} \\
\tau_{\mathrm{p}}=400 \mu \mathrm{s}\end{array}$} & \multicolumn{2}{|c|}{$\begin{array}{c}\text { FWHM }^{*} \\
\tau_{\mathrm{p}}=800 \mu \mathrm{s}\end{array}$} & \multicolumn{2}{|c|}{$\begin{array}{c}\text { FWHM }^{*} \\
\tau_{\mathrm{p}}=2000 \mu \mathrm{s} \\
\text { (centre) }\end{array}$} & \multicolumn{2}{|c|}{$\begin{array}{c}\mathrm{FWHM}^{*} \\
\tau_{\mathrm{p}}=2000 \mu \mathrm{s} \\
\quad(\text { edge })\end{array}$} \\
\hline & & & 240Н & & $230 \mathrm{~Hz}$ & & $200 \mathrm{~Hz}$ & & $200 \mathrm{~Hz}$ & \\
\hline & & & & & & & & & & \\
\hline 12.21 & $190 \mathrm{~Hz}$ & & $180 \mathrm{~Hz}$ & & $180 \mathrm{~Hz}$ & & $170 \mathrm{~Hz}$ & $0.50 \mathrm{ppm}$ & $200 \mathrm{~Hz}$ & $0.59 \mathrm{ppm}$ \\
\hline 16.79 & $240 \mathrm{~Hz}$ & $0.70 \mathrm{ppm}$ & $220 \mathrm{~Hz}$ & $0.63 \mathrm{ppm}$ & $190 \mathrm{~Hz}$ & $0.56 \mathrm{ppm}$ & $180 \mathrm{~Hz}$ & $0.52 \mathrm{ppm}$ & $230 \mathrm{~Hz}$ & $0.68 \mathrm{ppm}$ \\
\hline
\end{tabular}

* Values given in $\mathrm{Hz}$ are taken from the processed and unscaled spectra. Values in ppm include the isotropic chemical-shift scaling and correspond to the values in the plotted spectra.

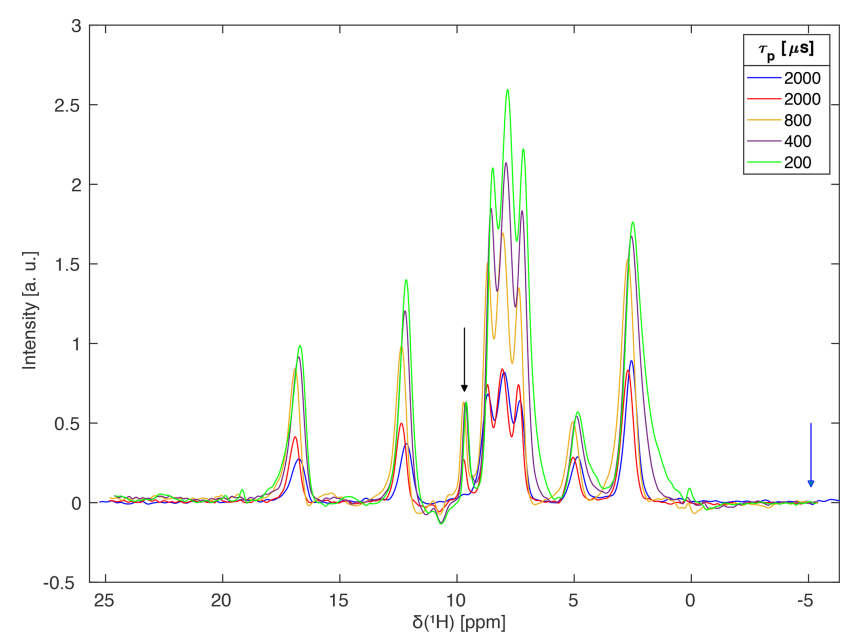

Figure 8. Homonuclear-decoupled proton spectra of L-histidine using FSLG decoupling and different selectivity of the I-BURP-2 pulse in the spin-lock frame at $14 \mathrm{kHz}$ MAS. The spectra were acquired at a proton resonance frequency of $600 \mathrm{MHz}$ using a Bruker $2.5 \mathrm{~mm}$ probe which has a much stronger rf-field inhomogeneity than the $1.9 \mathrm{~mm}$ MAS probe used in Fig. 7. The carrier was placed in the centre of the region of interest (arrow indicating the exact position) for all spectra except the blue one. One can clearly see that longer (more selective) pulses reduce the intensity of the signal but lead to narrower lines, especially on the side of the peak pointing away from the carrier frequency. There is a small carrier-frequency artefact for the spectra with the carrier frequency inside the spectral region of interest. Experimental scaling factors were 0.572 for the $2 \mathrm{~ms}$ I-BURP-2 pulses, 0.575 for the $800 \mu$ s pulse, and 0.579 for the 400 and $200 \mu$ s pulses. The modulation frequency was set to $100 \mathrm{kHz}$ for the $2 \mathrm{~ms}$ and $800 \mu \mathrm{s}, 95 \mathrm{kHz}$ for the $400 \mu \mathrm{s}$, and $90 \mathrm{kHz}$ for the $200 \mu$ s I-BURP-2 pulses. The modulation frequencies were selected such that the bandwidths of the different pulses cover the region of maximum intensity in the nutation spectrum.

formed at proton resonance frequencies of 500 and $600 \mathrm{MHz}$ at a spinning speed of $14 \mathrm{kHz}$. The rf-field amplitudes used for hard pulses and during spin lock were set to $100 \mathrm{kHz}$ as determined using a nutation experiment. Shaped pulses using a phase ramp with $50 \mathrm{~ns}$ time resolution were used for the implementation of FSLG decoupling. Each shape consists of 320 points leading to a total length of $16 \mu$ s which corresponds to a nutation frequency about the effective field of $125 \mathrm{kHz}$. Spectra were recorded with $350-768 t_{1}$ increments with 8 to 16 scans each and a time increment between 48 and $96 \mu$ s (spectral width 20.8 to $10.4 \mathrm{kHz}$ ). The spectral width in $t_{2}$ was set to $200 \mathrm{kHz}$ and 1024 complex data points were acquired. For most experiments, the carrier was set to the edge of the spectral region of interest, but no experimental optimization of the carrier position was performed. In all spectra, the carrier position is indicated by an arrow. A more detailed summary of the acquisition parameters used for the homonuclear-decoupled proton spectra can be found in Table 1 in the Supplement. For processing in Matlab, zero filling to $4096 \times 4096$ data points was used and a cosine-squared window function applied. The one-dimensional spectra were obtained by summing over the relevant spectral region in $\omega_{2}$. Experimental chemical shift scaling factors were determined by comparing the observed peak positions to those found in the literature. The reference peaks used for the comparison were the $\mathrm{NH}_{3}$ resonance and the centre of the methylene resonance ( 8 and $3.16 \mathrm{ppm}$ ) for glycine (Mote et al., 2016), the $\mathrm{OH}$ and $\mathrm{CH}_{3}$ resonances (12.85 and $0.86 \mathrm{ppm}$ ) for $\beta$-Asp-Ala (Paruzzo et al., 2019), and the $\alpha$ and $\delta^{2}$ proton resonances (16.75 and $2.55 \mathrm{ppm})$ for L-histidine $\cdot \mathrm{HCl} \cdot \mathrm{H}_{2} \mathrm{O}$ (Mithu et al., 2013).

\section{Conclusions and outlook}

We have shown that band-selective pulses in a spin-lock frame can be used to implement nutation-frequency selective pulses with very good excitation profiles. Implementing nutation-frequency selective pulses in this way offers a large range of possible pulse shapes and leads to more stable results than direct numerical optimization of these pulses described in the literature thus far (Charmont et al., 2002). Such $B_{1}$-field selective pulses can be used to reduce detrimental effects in pulse sequences that are sensitive to rf-field inhomogeneity and present a much simpler and more effective alternative to spatial sample restriction along the rotor axis. As an example, we show significant improvements in homonuclear-decoupled spectra under MAS using FSLG decoupling. On the one hand, the zero-frequency artefact is al- 
most eliminated and the lines are narrower due to the reduction of the rf-field inhomogeneity. At the same time, the intensity of the signal intensity is reduced, and a compromise between resolution and sensitivity has to be found. We believe that pulse lengths around $500 \mu$ s and $1 \mathrm{~ms}$ (bandwidth of 4 to $8 \mathrm{kHz}$ ) provide a good compromise. The implementation of the pulses is straightforward, and any inversion pulse shape can be used. In principle selective $90^{\circ}$ pulses or more complex composite pulses could also be used to implement a selection of $B_{1}$ fields or other manipulations of the magnetization in the spin-lock frame. Moreover, nutation-frequency selective pulses could also be used for probe background suppression (Feng and Reimer, 2011) in a simple difference experiment since the probe background typically experiences much lower rf-field amplitudes than the sample inside the coil.

Data availability. The experimental NMR data, the simulation data, and the processing and plot scripts for all figures are available at https://doi.org/10.3929/ethz-b-000432681 (Aebischer et al., 2020).

Supplement. The supplement related to this article is available online at: https://doi.org/10.5194/mr-1-187-2020-supplement.

Author contributions. NW and ME designed the research. ZT provided data about rf-field inhomogeneity in probes. KA and ME carried out all measurements and simulations. All the authors were involved in writing the manuscript.

Competing interests. The authors declare that they have no conflict of interest.

Special issue statement. This article is part of the special issue "Geoffrey Bodenhausen Festschrift". It is not associated with a conference.

Acknowledgements. We would like to thank Perunthiruthy K. Madhu, Kaustubh Mote, and Johannes Hellwagner for insightful discussions about theory and experimental implementation of homonuclear decoupling. Beat H. Meier and Alexander Barnes are acknowledged for providing measurement time for the project.

Financial support. This research has been supported by the Schweizerischer Nationalfonds zur Förderung der Wissenschaftlichen Forschung (grant no. 200020_188988) and the Czech Science Foundation (grant no. 20-00166J).
Review statement. This paper was edited by Konstantin Ivanov and reviewed by Paul Hodgkinson and one anonymous referee.

\section{References}

Aebischer, K., Wili, N., Tošner, Z., and Ernst, M.: Using nutation-frequency-selective pulses to reduce radio-frequency field inhomogeneity in solid-state NMR, ETH Zürich, https://doi.org/10.3929/ethz-b-000432681, 2020.

Bielecki, A., Kolbert, A., and Levitt, M. H.: Frequency-Switched Pulse Sequences - Homonuclear Decoupling and Dilute Spin NMR in Solids, Chem. Phys. Lett., 155, 341-346, 1989.

Bielecki, A., Kolbert, A., de Groot, H. J. M., and Griffin, R. G.: Frequency-switched Lee-Goldburg sequences in solids, Adv. Magn. Reson., 14, 111-124, 1990.

Bloch, F. and Siegert, A.: Magnetic resonance for nonrotating fields, Phys. Rev., 57, 522-527, 1940.

Charmont, P., Lesage, A., Steuernagel, S., Engelke, F., and Emsley, L.: Sample Restriction Using Magnetic Field Gradients in HighResolution Solid-State NMR, J. Magn. Reson., 145, 334-339, https://doi.org/10.1006/jmre.2000.2117, 2000.

Charmont, P., Sakellariou, D., and Emsley, L.: Sample restriction using radiofrequency field selective pulses in highresolution solid-state NMR, J. Magn. Reson., 154, 136-141, https://doi.org/10.1006/jmre.2001.2467, 2002.

Emsley, L.: Selective Pulses, in: eMagRes, edited by: Harris, R. K. and Wasylishen, R. E., John Wiley \& Sons, Ltd, Chichester, UK, 2007.

Ernst, R. R., Bodenhausen, G., and Wokaun, A.: Principles of Nuclear Magnetic Resonance in One and Two Dimensions, Oxford University Press on Demand, 1990.

Feng, J. and Reimer, J. A.: Suppression of probe background signals via $\mathrm{B}_{1}$ field inhomogeneity, J. Magn. Reson., 209, 300-305, https://doi.org/10.1016/j.jmr.2011.01.023, 2011.

Geen, H. and Freeman, R.: Band-Selective Radiofrequency Pulses, J. Magn. Reson., 93, 93-141, https://doi.org/10.1016/00222364(91)90034-Q, 1991.

Grzesiek, S. and Bax, A.: Audio-Frequency NMR in a Nutating Frame, Application to the Assignment of Phenylalanine Residues in Isotopically Enriched Proteins, J. Am. Chem. Soc., 117, 65276531, https://doi.org/10.1021/ja00129a016, 1995.

Guenneugues, M., Berthault, P., and Desvaux, H.: A method for determining B-1 field inhomogeneity. Are the biases assumed in heteronuclear relaxation experiments usually underestimated?, J. Magn. Reson., 136, 118-126, https://doi.org/10.1006/jmre.1998.1590, 1999.

Gupta, R., Hou, G., Polenova, T., and Vega, A. J.: RF inhomogeneity and how it controls CPMAS, Solid State Nucl. Magn. Reson., 72, 17-26, https://doi.org/10.1016/j.ssnmr.2015.09.005, 2015.

Hellwagner, J., Grunwald, L., Ochsner, M., Zindel, D., Meier, B. H., and Ernst, M.: Origin of the residual line width under frequencyswitched Lee-Goldburg decoupling in MAS solid-state NMR, Magn. Reson., 1, 13-25, https://doi.org/10.5194/mr-1-13-2020, 2020.

Khaneja, N. and Nielsen, N. C.: Triple oscillating field technique for accurate distance measurements by solid-state NMR, J. Chem. Phys., 128, 015103, https://doi.org/10.1063/1.2816140, 2008.

Lindon, J. C., Beckonert, O. P., Holmes, E., and Nicholson, J. K.: High-resolution magic angle spinning NMR spectroscopy: Ap- 
plication to biomedical studies, Prog. NMR Spectr., 55, 79-100, https://doi.org/10.1016/j.pnmrs.2008.11.004, 2009.

Mithu, V. S., Tan, K. O., and Madhu, P. K.: Selective inversion of $1 \mathrm{H}$ resonances in solid-state nuclear magnetic resonance: Use of double-DANTE pulse sequence, J. Magn. Reson., 237, 11-16, https://doi.org/10.1016/j.jmr.2013.09.010, 2013.

Mote, K. R., Agarwal, V., and Madhu, P. K.: Five decades of homonuclear dipolar decoupling in solid-state NMR: Status and outlook, Prog. NMR Spectr., 97, 1-39, https://doi.org/10.1016/j.pnmrs.2016.08.001, 2016.

Nishimura, K., Fu, R., and Cross, T. A.: The Effect of RF Inhomogeneity on Heteronuclear Dipolar Recoupling in Solid State NMR: Practical Performance of SFAM and REDOR, J. Magn. Reson., 152, 227-233, https://doi.org/10.1006/jmre.2001.2410, 2001.

Odedra, S. and Wimperis, S.: Imaging of the B1 distribution and background signal in a MAS NMR probehead using inhomogeneous B0 and B1 fields, J. Magn. Reson., 231, 95-99, https://doi.org/10.1016/j.jmr.2013.04.002, 2013.

Paruzzo, F. M., Walder, B. J., and Emsley, L.: Line narrowing in ${ }^{1} \mathrm{H}$ NMR of powdered organic solids with TOP-CT-MAS experiments at ultra-fast MAS, J. Magn. Reson., 305, 131-137, https://doi.org/10.1016/j.jmr.2019.06.015, 2019.

Paulson, E. K., Martin, R. W., and Zilm, K. W.: Cross polarization, radio frequency field homogeneity, and circuit balancing in high field solid state NMR probes, J. Magn. Reson., 171, 314-323, https://doi.org/10.1016/j.jmr.2004.09.009, 2004.

Smith, S. A., Levante, T. O., Meier, B. H., and Ernst, R. R.: Computer-Simulations in Magnetic-Resonance - an ObjectOriented Programming Approach, J. Magn. Reson. Ser. A, 106, 75-105, 1994.
States, D. J., Haberkorn, R. A., and Ruben, D. J.: A TwoDimensional Nuclear Overhauser Experiment with Pure Absorption Phase in 4 Quadrants, J. Magn. Reson., 48, 286-292, https://doi.org/10.1016/0022-2364(82)90279-7, 1982.

Straas $\varnothing$, L. A., Bjerring, M., Khaneja, N., and Nielsen, N. C.: Multiple-oscillating-field techniques for accurate distance measurements by solid-state NMR, J. Chem. Phys., 130, 225103, https://doi.org/10.1063/1.3147010, 2009.

Tosner, Z., Purea, A., Struppe, J. O., Wegner, S., Engelke, F., Glaser, S. J., and Reif, B.: Radiofrequency fields in MAS solid state NMR probes, J. Magn. Reson., 284, 20-32, https://doi.org/10.1016/j.jmr.2017.09.002, 2017.

Tosner, Z., Sarkar, R., Becker-Baldus, J., Glaubitz, C., Wegner, S., Engelke, F., Glaser, S. J., and Reif, B.: Overcoming Volume Selectivity of Dipolar Recoupling in Biological Solid-State NMR Spectroscopy, Angew. Chem. Int. Ed. Engl., 57, 14514-14518, https://doi.org/10.1002/anie.201805002, 2018.

Vega, A. J.: Controlling the effects of pulse transients and RF inhomogeneity in phase-modulated multiple-pulse sequences for homonuclear decoupling in solid-state proton NMR, J. Magn. Reson., 170, 22-41, https://doi.org/10.1016/j.jmr.2004.05.017, 2004.

Wili, N., Hintz, H., Vanas, A., Godt, A., and Jeschke, G.: Distance measurement between trityl radicals by pulse dressed electron paramagnetic resonance with phase modulation, Magn. Reson., 1, 75-87, https://doi.org/10.5194/mr-1-75-2020, 2020. 